FNT/T-2001/19

\title{
A Path Integral Way to Option Pricing
}

\author{
Guido Montagna $^{\mathrm{a}, \mathrm{b}}$ and Oreste Nicrosini $\mathrm{b}, \mathrm{a}$ \\ ${ }^{a}$ Dipartimento di Fisica Nucleare e Teorica, Università di Pavia \\ Via A. Bassi 6, 27100, Pavia, Italy \\ ${ }^{\mathrm{b}}$ Istituto Nazionale di Fisica Nucleare, sezione di Pavia \\ Via A. Bassi 6, 27100, Pavia, Italy \\ Nicola Moreni ${ }^{\mathrm{c}}$ \\ ${ }^{\mathrm{c}}$ Université Pierre et Marie Curie Paris 6, Place Jussieu \\ 75252 Paris Cedex 05, France
}

\begin{abstract}
An efficient computational algorithm to price financial derivatives is presented. It is based on a path integral formulation of the pricing problem. It is shown how the path integral approach can be worked out in order to obtain fast and accurate predictions for the value of a large class of options, including those with path-dependent and early exercise features. As examples, the application of the method to European and American options in the Black-Scholes model is illustrated. A particularly simple and fast semi-analytical approximation for the price of American options is derived. The results of the algorithm are compared with those obtained with the standard procedures known in the literature and found to be in good agreement.
\end{abstract}

Key words: Econophysics; Stochastic Processes; Path Integral; Financial Derivatives; Option Pricing.

PACS: 02.50.Ey - 05.10.Gg - 89.75.-k 


\section{Introduction}

The classical theory of option pricing is based on the results found in 1973 by Black and Scholes [1] and, independently, Merton [2]. Their pioneering work starts from the basic assumption that the asset prices follow the dynamics of a particular stochastic process (geometric Brownian motion), so that they have a lognormal distribution [3,4]. In the case of an efficient market with no arbitrage possibilities, no dividends and constant volatilities, they found that the price of each financial derivative is ruled by an ordinary partial differential equation, known as the Black-Scholes-Merton (BSM) formula. In the most simple case of a so-called European option, the BSM equation can be explicitly solved to obtain an analytical formula for the price of the option $[3,4]$. When we consider other financial derivatives, which are commonly traded in real markets and allow anticipated exercise and/or depend on the history of the underlying asset, the BSM formula fails to give an analytical result. Appropriate numerical procedures have been developed in the literature to price exotic financial derivatives with path-dependent features, as discussed in detail in Refs. $[3,5,6]$. The aim of this work is to provide a contribution to the problem of efficient option pricing in financial analysis, showing how it is possible to use path integral methods to develop a fast and precise algorithm for the evaluation of option prices.

The path integral method, which traces back to the original work of Wiener and Kac in stochastic calculus [7,8] and of Feynman in quantum mechanics [9], is today widely employed in chemistry and physics, and very recently in finance too [10-14], because it gives the possibility of applying powerful analytical and numerical techniques [15]. Following recent studies on the application of the path integral approach to the financial market as appeared in the econophysics literature (see Refs. [12,14] for a comprehensive list of references), this paper is devoted to present an original, efficient path integral algorithm to price financial derivatives, including those with path-dependent and early exercise features, and to compare the results with those obtained with the standard procedures known in the literature.

The paper is organized as follows. In Section 2 the basic ideas of the classical theory of option pricing are summarized, discussing the computational complexity associated to the evaluation of the price of a path-dependent option and reviewing the standard numerical procedures adopted in the literature. In Section 3 the path integral approach to option pricing is described and analytically developed in order to obtain an efficient procedure for the calculation of the transition probability associated to a given stochastic model of asset evolution. Theoretical and computational details to obtain fast predictions for path-dependent options are also described. As applications of the method, numerical results for European and American options in the BSM model are 
given in Section 4, together with comparisons with results known in the literature. A particularly simple and very quick semi-analytical approximation for the price of an American option is derived in Section 5, by exploiting the possibility of anticipated exercise for any time before the expiration date. Conclusions and possible perspectives are drawn in Section 6.

\section{Option pricing: theory and numerical procedures}

\subsection{Classical theory and path-dependent options}

The basic ingredient for the development of a theory of option pricing is a suitable model for the time evolution of the asset prices. The assumption of the BSM model is that the price $S$ of an asset is driven by a geometric Brownian motion and verifies the stochastic differential equation (SDE) [3,4]

$$
d S=\mu S d t+\sigma S d w
$$

which, by means of the Itô lemma, can be cast in the form of an arithmetic Brownian motion for the logarithm of $S$

$$
d(\ln S)=A d t+\sigma d w
$$

where $\sigma$ is the volatility, $A \doteq\left(\mu-\sigma^{2} / 2\right), \mu$ is the drift parameter and $w$ is the realization of a Wiener process. By virtue of the properties of a Wiener process, eq. (2) may be written as

$$
d(\ln S)=A d t+\sigma \epsilon \sqrt{d t}
$$

where $\epsilon$ follows from a standardized normal distribution with mean 0 and variance 1.

Thus, in terms of the logarithms of the asset prices $z^{\prime} \doteq \ln S^{\prime}, z \doteq \ln S$, the conditional transition probability $p\left(z^{\prime} \mid z\right)$ to have at the time $t^{\prime}$ a price $S^{\prime}$ under the hypothesis that the price was $S$ at the time $t<t^{\prime}$ is given by $[4,10] \square$

$$
p\left(z^{\prime} \mid z\right)=\frac{1}{\sqrt{2 \pi\left(t^{\prime}-t\right) \sigma^{2}}} \exp \left\{-\frac{\left[z^{\prime}-\left(z+A\left(t^{\prime}-t\right)\right)\right]^{2}}{2 \sigma^{2}\left(t^{\prime}-t\right)}\right\}
$$

$\overline{1}$ The correct way to indicate conditional transition probabilities is $p\left(z^{\prime}, t^{\prime} \mid z, t\right)$. We omit the times in order to simplify the notation. 
which is a gaussian distribution with mean $z+A\left(t^{\prime}-t\right)$ and variance $\sigma^{2}\left(t^{\prime}-t\right)$. If we require the options to be exercised only at specific times $t_{i}, i=1, \cdots, n$, the asset price, between two consequent times $t_{i-1}$ and $t_{i}$, will follow eq. (3) and the related transition probability will be

$$
p\left(z_{i} \mid z_{i-1}\right)=\frac{1}{\sqrt{2 \pi \Delta t \sigma^{2}}} \exp \left\{-\frac{\left[z_{i}-\left(z_{i-1}+A \Delta t\right)\right]^{2}}{2 \sigma^{2} \Delta t}\right\}
$$

with $\Delta t=t_{i}-t_{i-1}$.

A time-evolution model for the asset price is strictly necessary in a theory of option pricing because the fair price at time $t=0$ of an option $\mathcal{O}$, without possibility of anticipated exercise before the expiration date or maturity $T$ (a so-called European option), is given by the scaled expectation value [3]

$$
\mathcal{O}(0)=e^{-r T} E[\mathcal{O}(T)]
$$

where $r$ is the risk-free interest and $E[\cdot]$ indicates the mean value, which can be computed only if a model for the asset underlying the option is understood. For example, the value $\mathcal{O}$ of an European call option at the maturity $T$ will be $\max \left\{S_{T}-X, 0\right\}$, where $X$ is the strike price, while for an European put option the value $\mathcal{O}$ at the maturity will be $\max \left\{X-S_{T}, 0\right\}$. It is worth emphasizing, for what follows, that the case of an European option is particularly simple, since in such a situation the price of the option can be evaluated by means of analytical formulae, which are obtained by solving the BSM partial differential equation with the appropriate boundary conditions $[3,4]$. On the other hand, many further kinds of options are present in the financial markets, such as American options (options which can be exercised at any time up to the expiration date) and exotic options [3], i.e. derivatives with complicated payoffs or whose value depend on the whole time evolution of the underlying asset and not just on its value at the end. For such options with path-dependent and early exercise features no exact solutions are available and pricing them correctly is a great challenge.

Actually, in the case of options with possibility of anticipated exercise before the expiration date, the above discussion needs to be generalized, by introducing a slicing of the time interval $T$. Let us consider, for definiteness, the case of an option which can be exercised within the maturity but only at the times $t_{1}=\Delta t, t_{2}=2 \Delta t, \ldots, t_{n}=n \Delta t \doteq T$. At each time slice $t_{i-1}$ the value $\mathcal{O}_{i-1}$ of the option will be the maximum between its expectation value at the time $t_{i}$ scaled with $e^{-r \Delta t}$ and its value in the case of anticipated exercise $\mathcal{O}_{i-1}^{Y}{ }^{2}$. If $S_{i-1}$ denotes the price of the underlying asset at the time $t_{i-1}$, we can thus

2 For example, the value $\mathcal{O}$ of a call option in the case of exercise at the time $t_{i-1}$ will be $\max \left\{S_{i-1}-X, 0\right\}, X$ being the strike price. 
write for each $i=1, \ldots, n$

$$
\mathcal{O}_{i-1}\left(S_{i-1}\right)=\max \left\{\mathcal{O}_{i-1}^{Y}\left(S_{i-1}\right), e^{-r \Delta t} E\left[\mathcal{O}_{i} \mid S_{i-1}\right]\right\}
$$

where $E\left[\mathcal{O}_{i} \mid S_{i-1}\right]$ is the conditional expectation value of $\mathcal{O}_{i}$, i.e. its expectation value under the hypothesis of having the price $S_{i-1}$ at the time $t_{i-1}$. In this way, to get the actual price $\mathcal{O}_{0}$, it is necessary to proceed backward in time and calculate $\mathcal{O}_{n-1}, \ldots, \mathcal{O}_{1}$, where the value $\mathcal{O}_{n}$ of the option at maturity is nothing but $\mathcal{O}_{n}^{Y}\left(S_{n}\right)$. It is therefore clear that evaluating the price of an option with early exercise features means to simulate the evolution of the underlying asset price (to obtain the $\mathcal{O}_{i}^{Y}$ ) and to calculate a (usually large) number of expectation conditional probabilities.

\subsection{Standard numerical procedures}

To value derivatives when analytical formulae are not available, appropriate numerical techniques have to be advocated. They involve the use of Monte Carlo (MC) simulation, binomial trees (and their improvements) and finite difference methods $[3,5]$.

A natural way to simulate price paths is to discretize eq. (3) as

$$
\ln S(t+\Delta t)-\ln S(t)=A \Delta t+\sigma \epsilon \sqrt{\Delta t}
$$

or, equivalently,

$$
S(t+\Delta t)=S(t) \exp [A \Delta t+\sigma \epsilon \sqrt{\Delta t}]
$$

which is correct for any $\Delta t>0$, even if finite. Given the spot price $S_{0}$, i.e. the price of the asset at time $t=0$, one can extract from a standardized normal distribution a value $\epsilon_{k}, k=1, \ldots, n$ for the random variable $\epsilon$ to simulate one possible path followed by the price by means of eq. (8):

$$
S(k \Delta t)=S((k-1) \Delta t) \exp \left[A \Delta t+\sigma \epsilon_{k} \sqrt{\Delta t}\right]
$$

Iterating the procedure $m$ times, one can simulate $m$ price paths $\left\{\left(S_{0}, S_{1}^{(j)}, S_{2}^{(j)}\right.\right.$, $\left.\left.\ldots, S_{n}^{(j)} \equiv S_{T}^{(j)}\right): j=1, \ldots, m\right\}$, to which apply the procedure exemplified in Section 2.1 and evaluate the price of the option. In such a MC simulation of 
the stochastic dynamics of asset price (Monte Carlo random walk) the mean values $E\left[\mathcal{O}_{i} \mid S_{i-1}\right], i=1, \ldots, n$ can be simply obtained as

$$
E\left[\mathcal{O}_{i} \mid S_{i-1}\right]=\frac{\mathcal{O}_{i}^{(1)}+\mathcal{O}_{i}^{(2)}+\cdots+\mathcal{O}_{i}^{(m)}}{m}
$$

with no need to calculate transition probabilities because, through the extraction of the possible $\epsilon$ values, the paths are automatically weighted according to the probability distribution function of eq. (5).

Unfortunately, this method leads to an estimated value whose numerical error is proportional to $m^{-1 / 2}$. Thus, even if it is powerful because of the possibility to control the paths and to impose additional constrains (as it is usually required by exotic and path-dependent options), the MC random walk is extremely time consuming when precise predictions are required and appropriate variance reduction procedures have to be used to save CPU time [3].

This difficulty can be overcome by means of the method of the binomial trees and its extensions (see [3] and references therein), whose main idea stands in a deterministic choice of the possible paths to limit the number of intermediate points. At each time step the price $S_{i}$ is assumed to have only two choices: increase to the value $u S_{i}, u>1$ or decrease to $d S_{i}, 0<d<1$, where the parameters $u$ and $d$ are given in terms of $\sigma$ and $\Delta t$ in such a way to give the correct values for the mean and variance of stock price changes over the time interval $\Delta t$. Also finite difference methods are known in the literature [3] as an alternative to time-consuming MC simulations. They provide the value of the derivative by solving the differential equation satisfied by the derivative, by converting it into a difference equation. Although tree approaches and finite difference methods are known to be faster than the MC random walk, they are difficult to apply when a detailed control of the history of the derivative is required and are also computationally time consuming when a number of stochastic variables is involved [3]. It follows that the development of efficient and fast computational algorithms to price financial derivatives is still a key issue in financial analysis.

\section{The path integral method}

The path integral method is an integral formulation of the dynamics of a stochastic process. It is a suitable framework for the calculation of the transition probabilities associated to a given stochastic process, which is seen as the convolution of an infinite sequence of infinitesimal short-time steps $[10,15]$. For the problem of option pricing, the path integral method can be employed for the explicit calculation of the expectation values of the quantities of finan- 
cial interest, given by integrals of the form [10]

$$
E\left[\mathcal{O}_{i} \mid S_{i-1}\right]=\int d z_{i} p\left(z_{i} \mid z_{i-1}\right) \mathcal{O}_{i}\left(e^{z_{i}}\right)
$$

where $z=\ln S$ and $p\left(z_{i} \mid z_{i-1}\right)$ is the transition probability. $E\left[\mathcal{O}_{i} \mid S_{i-1}\right]$ is the conditional expectation value of some functional $\mathcal{O}_{i}$ of the stochastic process. For example, for an European call option at the maturity $T$ the quantity of interest will be $\max \left\{S_{T}-X, 0\right\}, X$ being the strike price. As already emphasized, and discussed in the literature $[3,5,6,11,14]$, the computational complexity associated to this calculation is generally great: in the case of exotic options, with path-dependent and early exercise features, integrals of the type (9) can not be analytically solved. As a consequence, we demand two things from a path integral framework: a very quick way to estimate the transition probability associated to a stochastic process (3) and a clever choice of the integration points with which evaluate the integrals (9). In particular, our aim is to develop an efficient calculation of the probability distribution without losing information on the path followed by the asset price during its time evolution.

\subsection{Transition probability}

The probability distribution function related to a SDE verifies the so-called Chapman-Kolmogorov equation [4], i.e. the relation

$$
p\left(z^{\prime \prime} \mid z^{\prime}\right)=\int d z p\left(z^{\prime \prime} \mid z\right) p\left(z \mid z^{\prime}\right)
$$

which states that the probability (density) of a transition from the value $z^{\prime}$ (at time $t^{\prime}$ ) to the value $z^{\prime \prime}$ (at time $t^{\prime \prime}$ ) is the "summation" over all the possible intermediate values $z$ of the probability of separate and consequent transitions $z^{\prime} \rightarrow z, z \rightarrow z^{\prime \prime}$.

As a consequence, if we consider a finite time interval $\left[t^{\prime}, t^{\prime \prime}\right]$ and we apply a time slicing, by considering $n+1$ subintervals of length $\Delta t \doteq\left(t^{\prime \prime}-t^{\prime}\right) / n+1$, we can write, by iteration of eq. (10)

$$
p\left(z^{\prime \prime} \mid z^{\prime}\right)=\int_{-\infty}^{+\infty} \cdots \int_{-\infty}^{+\infty} d z_{1} \cdots d z_{n} p\left(z^{\prime \prime} \mid z_{n}\right) p\left(z_{n} \mid z_{n-1}\right) \cdots p\left(z_{1} \mid z^{\prime}\right)
$$


which, thanks to eq.(4), can be written as

$$
\int_{-\infty}^{+\infty} \cdots \int_{-\infty}^{+\infty} d z_{1} \cdots d z_{n} \frac{1}{\sqrt{\left(2 \pi \sigma^{2} \Delta t\right)^{n+1}}} \cdot \exp \left\{-\frac{1}{2 \sigma^{2} \Delta t} \sum_{k=1}^{n+1}\left[z_{k}-\left(z_{k-1}+A \Delta t\right)\right]^{2}\right\} .
$$

In the limit $n \rightarrow \infty, \Delta t \rightarrow 0$ such that $(n+1) \Delta t=\left(t^{\prime \prime}-t^{\prime}\right)$ (infinite sequence of infinitesimal time steps), the expression (11), as explicitly shown in Ref. [10], exhibits a Lagrangian structure and it is possible to express the transition probability in the path integral formalism as a convolution of the form [10]

$$
p\left(z^{\prime \prime}, t^{\prime \prime} \mid z^{\prime}, t^{\prime}\right)=\int_{\mathcal{C}} \mathcal{D}\left[\sigma^{-1} \tilde{z}\right] \exp \left\{-\int_{t^{\prime}}^{t^{\prime \prime}} L(\tilde{z}(\tau), \dot{\tilde{z}}(\tau) ; \tau) d \tau\right\}
$$

where $L$ is the Lagrangian

$$
L(\tilde{z}(\tau), \dot{\tilde{z}}(\tau) ; \tau)=\frac{1}{2 \sigma^{2}}[\dot{\tilde{z}}(\tau)-A]^{2}
$$

and the integral is performed (with functional measure $\mathcal{D}[\cdot]$ ) over the paths $\tilde{z}(\cdot)$ belonging to $\mathcal{C}$, i.e. all the continuous functions with constrains $\tilde{z}\left(t^{\prime}\right) \equiv z^{\prime}$, $\tilde{z}\left(t^{\prime \prime}\right) \equiv z^{\prime \prime}$. As carefully discussed in Ref. [10], a path integral is well defined only if both a continuous formal expression and a discretization rule are given. As done in many applications, the Itô prescription is adopted in the present work.

A first, naïve evaluation of the transition probability (11) can be performed via Monte Carlo simulation, by writing eq. (11) as

$$
p\left(z^{\prime \prime}, t^{\prime \prime} \mid z^{\prime}, t^{\prime}\right)=\int_{-\infty}^{+\infty} \cdots \int_{-\infty}^{+\infty} \prod_{i}^{n} d g_{i} \frac{1}{\sqrt{2 \pi \sigma^{2} \Delta t}} \exp \left\{-\frac{1}{2 \sigma^{2} \Delta t}\left[z^{\prime \prime}-\left(z_{n}+A \Delta t\right)\right]^{2}\right\}
$$

in terms of the variables $g_{i}$ defined by the relation

$$
d g_{k} \doteq \frac{d z_{k}}{\sqrt{2 \pi \sigma^{2} \Delta t}} \exp \left\{-\frac{1}{2 \sigma^{2} \Delta t}\left[z_{k}-\left(z_{k-1}+A \Delta t\right)\right]^{2}\right\}
$$

and extracting each $g_{i}$ from a gaussian distribution of mean $z_{k-1}+A \Delta t$ and variance $\sigma^{2} \Delta t$. However, as we will see, this method requires a large number of calls to obtain a good precision. This is due to the fact that each $g_{i}$ is related to the previous $g_{i-1}$, so that this implementation of the path integral approach can be seen to be equivalent to a naïve $\mathrm{MC}$ simulation of random walks, with no variance reduction. 
By means of appropriate manipulations [15] of the integrand entering eq. (11), it is possible, as shown in the following, to obtain a path integral expression which will contain a factorized integral with a constant kernel and a consequent variance reduction. We will refer to this second implementation of the method as path integral with importance sampling.

If we define $z^{\prime \prime} \doteq z_{n+1}$ and $y_{k} \doteq z_{k}-k A \Delta t, k=1, \ldots, n$, we can express the transition probability distribution as

$$
\int_{-\infty}^{+\infty} \cdots \int_{-\infty}^{+\infty} d y_{1} \cdots d y_{n} \frac{1}{\sqrt{\left(2 \pi \sigma^{2} \Delta t\right)^{n+1}}} \cdot \exp \left\{-\frac{1}{2 \sigma^{2} \Delta t} \sum_{k=1}^{n+1}\left[y_{k}-y_{k-1}\right]^{2}\right\}
$$

in order to get rid of the contribution of the drift parameter. Now let us extract from the argument of the exponential function a quadratic form

$$
\begin{gathered}
\sum_{k=1}^{n+1}\left[y_{k}-y_{k-1}\right]^{2}=y_{0}^{2}-2 y_{1} y_{0}+y_{1}^{2}+y_{1}^{2}-2 y_{1} y_{2}+\ldots+y_{n+1}^{2}= \\
=y^{t} M y+\left[y_{0}^{2}-2 y_{1} y_{0}+y_{n+1}^{2}-2 y_{n} y_{n+1}\right]
\end{gathered}
$$

by introducing the $n$-dimensional array $y$ and the $n \times n$ matrix $M$ defined as

$$
y=\left(\begin{array}{l}
y_{1} \\
y_{2} \\
\vdots \\
\vdots \\
y_{n}
\end{array}\right), \quad M=\left(\begin{array}{cccccc}
2 & -1 & 0 & \cdots & \cdots & 0 \\
-1 & 2 & -1 & 0 & \cdots & 0 \\
0 & -1 & 2 & -1 & \cdots & 0 \\
0 & \ldots & -1 & 2 & -1 & 0 \\
0 & \ldots & \ldots & -1 & 2 & -1 \\
0 & \ldots & \ldots & \ldots & -1 & 2
\end{array}\right)
$$

where $M$ is a real, symmetric, non singular and tridiagonal matrix. In terms of the eigenvalues $m_{i}$ of the matrix $M$, the contribution in eq. (15) can be written as

$$
y^{t} M y=w^{t} O^{t} M O w=w^{t} M_{d} w=\sum_{i=1}^{n} m_{i} w_{i}^{2},
$$

by introducing the orthogonal matrix $O$ which diagonalizes $M$, with $w_{i}=$ $O_{i j} y_{j}$. Because of the orthogonality of $O$, the Jacobian

$$
J=\operatorname{det}\left|\frac{d w_{i}}{d y_{k}}\right|=\operatorname{det}\left|O_{k i}\right|
$$


of the transformation $y_{k} \rightarrow w_{k}$ equals 1 , so that $\prod_{i=1}^{n} d w_{i}=\prod_{i=1}^{n} d y_{i}$. Thanks to eqs. (16)-(17), and after some algebra, eq. (15) can be written as

$$
\begin{gathered}
\sum_{k=1}^{n+1}\left[y_{k}-y_{k-1}\right]^{2}=\sum_{i=1}^{n} m_{i} w_{i}^{2}+y_{0}^{2}-2 y_{1} y_{0}+y_{n+1}^{2}-2 y_{n} y_{n+1}= \\
\sum_{i=1}^{n} m_{i}\left[w_{i}-\frac{\left(y_{0} O_{1 i}+y_{n+1} O_{n i}\right)}{m_{i}}\right]^{2}+y_{0}^{2}+y_{n+1}^{2}-\sum_{i=1}^{n} \frac{\left(y_{0} O_{1 i}+y_{n+1} O_{n i}\right)^{2}}{m_{i}} .
\end{gathered}
$$

Now, if we introduce new variables $h_{i}$ obeying the relation

$$
d h_{i} \doteq \sqrt{\frac{m_{i}}{2 \pi \sigma^{2} \Delta t}} \exp \left\{-\frac{m_{i}}{2 \sigma^{2} \Delta t}\left[w_{i}-\frac{\left(y_{0} O_{1 i}+y_{n+1} O_{n i}\right)}{m_{i}}\right]^{2}\right\} d w_{i}
$$

it is possible to express the finite-time probability distribution $p\left(z^{\prime \prime} \mid z^{\prime}\right)$ as

$$
\begin{gathered}
\int_{-\infty}^{+\infty} \cdots \int_{-\infty}^{+\infty} \prod_{i=1}^{n} d y_{i} \frac{1}{\sqrt{\left(2 \pi \sigma^{2} \Delta t\right)^{n+1}}} \exp \left\{-\frac{1}{2 \sigma^{2} \Delta t} \sum_{k=1}^{n+1}\left[y_{k}-y_{k-1}\right]^{2}\right\}= \\
=\int_{-\infty}^{+\infty} \cdots \int_{-\infty}^{+\infty} \prod_{i=1}^{n} d w_{i} \frac{1}{\sqrt{\left(2 \pi \sigma^{2} \Delta t\right)^{n+1}}} e^{-\left(y_{0}^{2}+y_{n+1}^{2}\right) / 2 \sigma^{2} \Delta t} \cdot \\
\cdot \exp \left\{-\frac{1}{2 \sigma^{2} \Delta t} \sum_{i=1}^{n}\left[m_{i}\left(w_{i}-\frac{\left(y_{0} O_{1 i}+y_{n+1} O_{n i}\right)}{m_{i}}\right)^{2}-\frac{\left(y_{0} O_{1 i}+y_{n+1} O_{n i}\right)^{2}}{m_{i}}\right]\right\}= \\
\int_{-\infty}^{+\infty} \ldots \int_{-\infty}^{+\infty} \prod_{i=1}^{n} d h_{i} \frac{1}{\sqrt{2 \pi \sigma^{2} \Delta t \operatorname{det}(M)}} \cdot \exp \left\{-\frac{1}{2 \sigma^{2} \Delta t}\left[y_{0}^{2}+y_{n+1}^{2}+\sum_{i=1}^{n} \frac{\left(y_{0} O_{1 i}+y_{n+1} O_{n i}\right)^{2}}{m_{i}}\right]\right\} \cdot(
\end{gathered}
$$

Equation (20) is one of the main results of the present work. Actually, the probability distribution function, as given by eq. (20), is an integral whose kernel is a constant function (with respect to the integration variables) and which can be factorized into the $n$ integrals

$$
\int_{-\infty}^{+\infty} d h_{i} \exp \left\{-\frac{1}{2 \sigma^{2} \Delta t} \frac{\left(y_{0} O_{1 i}+y_{n+1} O_{n i}\right)^{2}}{m_{i}}\right\}
$$

given in terms of the $h_{i}$, which are gaussian variables that can be extracted from a normal distribution with mean $\left(y_{0} O_{1 i}+y_{n+1} O_{n i}\right)^{2} / m_{i}$ and variance $\sigma^{2} \Delta t / m_{i}$. Differently to the first, naïve implementation of the path integral, now each $h_{i}$ is no longer dependent on the previous $h_{i-1}$, and importance 
sampling over the paths is automatically accounted for.

It is worth noticing that, by means of the extraction of the random variables $h_{i}$, we are creating price paths, since at each intermediate time $t_{i}$ the asset price is given by

$$
S_{i}=\exp \left\{\sum_{k=1}^{n} O_{i k} h_{k}+i A \Delta t\right\}
$$

Therefore, the path integral algorithm can be easily adapted to the cases in which the derivative to be valued has, in the time interval $[0, T]$, additional constraints, as in the case of interesting path-dependent options, such as Asian and barrier options [3].

The results of the two realizations of the path integral method here discussed will be compared in Section 4.

\subsection{Integration points}

Thanks to the method illustrated in Section 3.1, a powerful and fast tool to compute the transition probability in the path integral framework is available and it can be employed if we need to value a generic option with maturity $T$ and with possibility of anticipated exercise at times $t_{i}=i \Delta t(n \Delta t \doteq T)$. As a consequence of this time slicing, one must numerically evaluate $n-1$ mean values of the type (9), in order to check at any time $t_{i}$, and for any value of the stock price, whether early exercise is more convenient with respect to holding the option for a future time. To keep under control the computational complexity and the time of execution, it is mandatory to limit as far as possible the number of points for the integral evaluation. This means that we would like to have a linear growth of the number of integration points with the time. Let us suppose to evaluate each mean value

$$
E\left[\mathcal{O}_{i} \mid S_{i-1}\right]=\int d z_{i} p\left(z_{i} \mid z_{i-1}\right) \mathcal{O}_{i}\left(e^{z_{i}}\right)
$$

with $p$ integration points, i.e. considering only $p$ fixed values for $z_{i}$. To this end, we can create a grid of possible prices, according to the dynamics of the stochastic process as given by eq. (3)

$$
z(t+\Delta t)-z(t)=\ln S(t+\Delta t)-\ln S(t)=A \Delta t+\epsilon \sigma \sqrt{\Delta t}
$$


Starting from $z_{0}$, we thus evaluate the expectation value $E\left[\mathcal{O}_{1} \mid S_{0}\right]$ with $p=$ $2 m+1, m \in \mathbb{N}$ values of $z_{1}$ centered 3 on the mean value $E\left[z_{1}\right]=z_{0}+A \Delta t$ and which differ from each other of a quantity of the order of $\sigma \sqrt{\Delta t}$

$$
z_{1}^{j} \doteq z_{0}+A \Delta t+j \sigma \sqrt{\Delta t}, \quad j=-m, \ldots,+m
$$

Going on like this, we can evaluate each expectation value $E\left[\mathcal{O}_{2} \mid z_{1}^{j}\right]$ obtained from each one of the $z_{1}$ 's created above with $p$ values for $z_{2}$ centered around the mean value

$$
E\left[z_{2} \mid z_{1}^{j}\right]=z_{1}^{j}+A \Delta t=z_{0}+2 A \Delta t+j \sigma \sqrt{\Delta t}
$$

Iterating the procedure until the maturity, we create a deterministic grid of points such that, at a given time $t_{i}$, there are $(p-1) i+1$ values of $z_{i}$, in agreement with the request of linear growth.

This procedure of selection of integration points, together with the calculation of the transition probability previously described, is the basis of our path integral simulation of the price of a generic option.

\section{Numerical results and discussion}

By applying the results derived in Section 3, we have at disposal an efficient path integral algorithm both for the calculation of transition probabilities and the evaluation of option prices. In the present section, the application of the method to European and American options in the BSM model is illustrated and comparisons with the results obtained with the standard procedures known in the literature are shown.

First, the path integral simulation of the probability distribution of the logarithm of the stock prices, $p(\ln S)$, as a function of the logarithm of the stock price, for a BSM-like stochastic model, as given by eq. (2), is shown in Fig. 1. The parameters used in the simulation are: $S_{0}=100, X=110, \mu=0.05$, $\sigma=0.1, t=0$ year and $T=1$ year, with 100 time slices. As can be seen, the expected lognormal distribution of the stock prices is correctly reproduced by the path integral numerical simulation. The plot shows a comparison of the calculation of $p(\ln S)$ as obtained by means of the two path integral algorithms described in Section 3.1. The markers correspond to the naïve path integral computation of the probability distribution, without variance reduction, for $10^{3}$ (upper plot) and $10^{4}$ (lower plot) MC iterations. The error bars indicate

$\overline{3}$ Let us recall that between two possible exercise times the probability distribution function is gaussian and it is therefore symmetrical with respect to its mean value. 

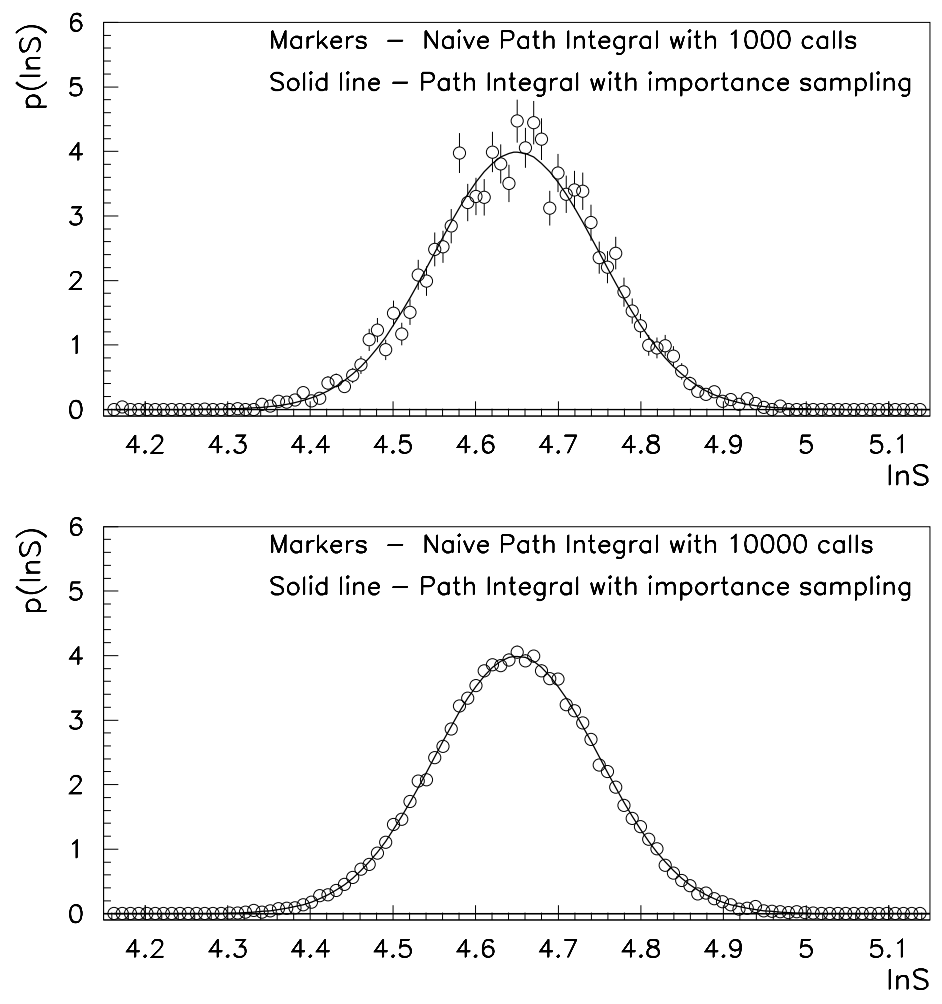

Fig. 1. Simulation of the transition probability distribution in the BSM model as a function of the logarithm of stock prices via the two path integral methods discussed in the text: the naïve path integral implementation for $10^{3}$ and $10^{4}$ Monte Carlo calls (markers) is compared with the path integral implementation with importance sampling (solid line).

the $1 \sigma$ statistical error of the MC calculation. The solid line is the prediction for $p(\ln S)$ as obtained with the path integral simulation with importance sampling. In such a case, only two calls for each variables $h_{i}$ are needed to correctly fit a gaussian distribution, the numerical error being totally negligible and the algorithm very fast, with a typical execution time of a few seconds on a PentiumIII 500 Mhz PC. On the contrary, the first path integral implementation is much less accurate and CPU time consuming. This is a consequence of the fact that, in the path integral simulation with importance sampling, the presence of constant integration kernel squeezes to zero the standard estimation error. The diagonalization of the tridiagonal matrix $M$, which is a basic ingredient of the efficient path integral algorithm developed, is performed according to the standard numerical procedure described in Ref. [16], realized by means of the routine F02FAF of the NAG program library [17], while the generation of the gaussian variables $h_{i}$ follows from the routine RNORML of the CERN program library.

As previously emphasized, the knowledge of the asset price $S_{i}$ at each intermediate time $t_{i}$ through eq. (22) gives the possibility of applying the procedure 
Table 1

Price of an European put option in the BSM model for the parameters $t=0$ year, $T=0.5$ year, $r=0.1, \sigma=0.4, X=10$, as a function of different stock prices $S_{0}$. 100 time slices are used in the path integral simulation.

\begin{tabular}{ccccc}
\hline \hline$S_{0}$ & analytical & binomial tree & GFDNM & path integral \\
\hline \hline 6.0 & 3.558 & 3.557 & 3.557 & 3.558 \\
8.0 & 1.918 & 1.917 & 1.917 & 1.918 \\
10.0 & 0.870 & 0.866 & 0.871 & 0.870 \\
12.0 & 0.348 & 0.351 & 0.349 & 0.348 \\
14.0 & 0.128 & 0.128 & 0.129 & 0.128 \\
\hline \hline
\end{tabular}

of calculation of the transition probability illustrated in Section 3.1 also to those types of financial derivatives whose payoff depends on the price path of the underlying asset. For such exotic options, the fast computation of the transition probability is a basic ingredient of our path integral algorithm as an efficient alternative to time-consuming $\mathrm{MC}$ simulation.

Once the transition probability has been computed, the price of an option can be computed in a path integral approach as the conditional expectation value of a given functional of the stochastic process. For example, the price of an European call option will be given by

$$
\mathcal{C}=e^{-r(T-t)} \int_{-\infty}^{+\infty} d z_{f} p\left(z_{f}, T \mid z_{i}, t\right) \max \left[e^{z_{f}}-X, 0\right]
$$

while for an European put it will be

$$
\mathcal{P}=e^{-r(T-t)} \int_{-\infty}^{+\infty} d z_{f} p\left(z_{f}, T \mid z_{i}, t\right) \max \left[X-e^{z_{f}}, 0\right]
$$

where $r$ is the risk-free interest rate. Therefore just one-dimensional integrals need to be evaluated. They can be precisely computed with standard quadrature rules. In our calculation, the one-dimensional integrals are simply performed with a standard trapezoidal rule, cross-checked with the routine of adaptive integration D01EAF from the NAG library [17]. A sample of the results obtained for an European put option in the BSM model is shown in Tab. 1. The predictions of our approach, indicated as path integral, are compared with results available in the literature, as quoted in Ref. [11]. In Tab. 1, the entries correspond to the analytical results, the results by binomial trees, and the results of the Green Function Deterministic Numerical Method (GFNDM) developed in Ref. [11]. As can be noticed, our results are in per- 

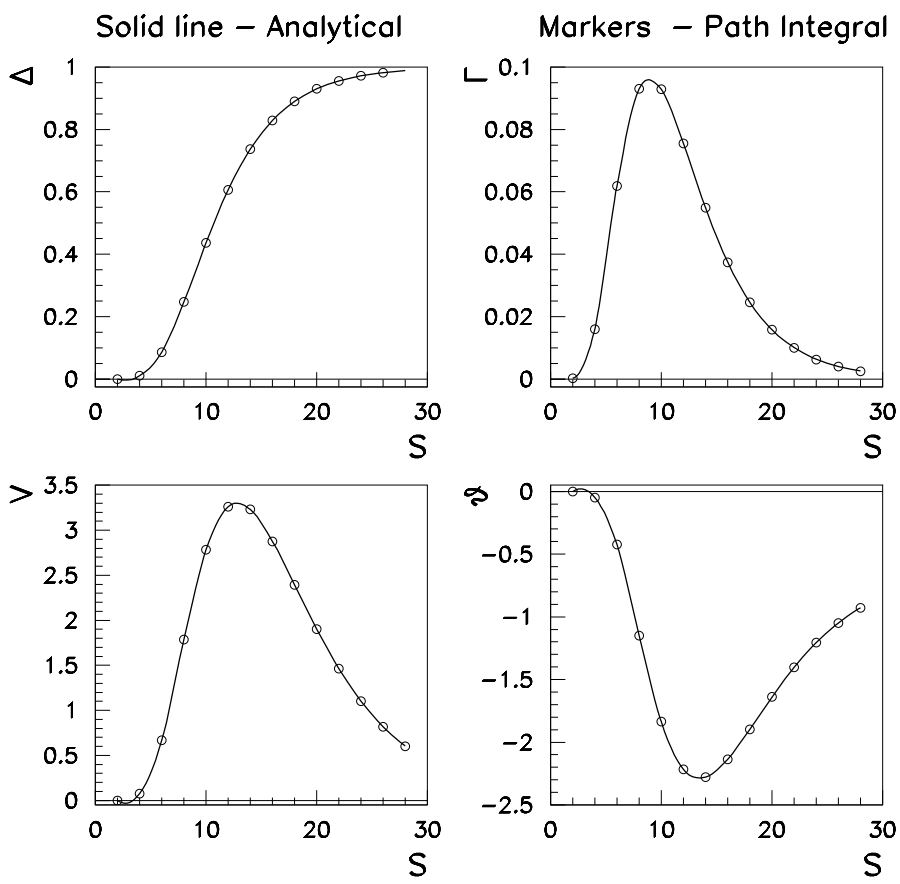

Fig. 2. The Greek letters delta $(\Delta)$, gamma $(\Gamma)$, vega $(V)$ and theta $(\vartheta)$ for an European call option in the BSM model, as functions of the stock price. The lines are the analytical predictions, while the markers are the results of our path integral algorithm.

fect agreement with the analytical predictions, while the differences with the other numerical procedures are within the $1 \%$ level. The errors in our numbers as due to numerical integration are not specified being well below the digits quoted.

Further numerical results of our path integral method are shown in Fig. 2, where the behaviour of the Greek letters [3] of an European call option in the BSM model is plotted as a function of the stock price. The solid lines correspond to the analytical predictions, while the markers are the results by our path integral approach. The model parameters used in the simulation are: $X=10, r=0.1, \sigma=0.4, t=0$ year and $T=0.5$ year. The four Greeks shown are important variables to manage the risk associated to an option and are defined as [3]

$$
\Delta=\frac{\partial \mathcal{C}}{\partial S}, \quad \Gamma=\frac{\partial^{2} \mathcal{C}}{\partial S^{2}}, \quad V=\text { Vega }=\frac{\partial \mathcal{C}}{\partial \sigma}, \quad \vartheta=\frac{\partial \mathcal{C}}{\partial t}
$$

where $\mathcal{C}$ is the price of the European call option. They are computed through 
Table 2

Price of an American put option in the BSM model for the parameters $t=0$ year, $T=0.5$ year, $r=0.1, \sigma=0.4, X=10$, as a function different stock prices $S_{0}$. The path integral simulation is performed with 200 time slicings and $p=13$ integration points.

\begin{tabular}{ccccc}
\hline \hline$S_{0}$ & finite difference & binomial tree & GFDNM & path integral \\
\hline \hline 6.0 & 4.00 & 4.00 & 4.00 & 4.00 \\
8.0 & 2.095 & 2.096 & 2.093 & 2.095 \\
10.0 & 0.921 & 0.920 & 0.922 & 0.922 \\
12.0 & 0.362 & 0.365 & 0.364 & 0.362 \\
14.0 & 0.132 & 0.133 & 0.133 & 0.132 \\
\hline \hline
\end{tabular}

standard numerical differentiation of the numerical value $\mathcal{C}$ returned by the path integral, by means of the NAG routine D04AAF [17]. Also for the Greeks, there is perfect agreement between the results obtained via our path integral simulation and the analytical predictions.

To test the reliability of the sampling over the integration points discussed in Section 3.2, we present results for the particular case of the price of an American option in the BSM model in Tab. 2, as obtained with the grid technique described in Section 3.2. Comparisons with independent results available in the literature are also shown. As can be seen from Tab. 2, there is generally a good agreement of our path integral results with those known in the literature [11] and obtained by means of the binomial tree, of the finite difference method and of the GFDNM method. It is worth noticing that our results in the path integral framework require only a few seconds on a PentiumIII 500MhZ PC.

\section{The limit of continuum and American options}

In the case of an American option, the possibility of exercise at any time up to the expiration date allows to develop, within the path integral formalism, a specific algorithm, which, as shown in the following, is precise and very quick. Given the time slicing considered in Section 3.2, the case of American options requires the limit $\Delta t \rightarrow 0$ which, putting $\sigma \rightarrow 0$, leads to a delta-like transition probability

$$
p\left(z, t+\Delta t \mid z_{t}, t\right) \approx \delta\left(z-z_{t}-A \Delta t\right)
$$


This means that, apart from volatility effects, the price $z_{i}$ at time $t_{i}$ will have a value remarkably close to the expected value $\bar{z} \doteq z_{i-1}+A \Delta t$, given by the drift growth. Needless to say, if we should substitute the expression $p\left(z_{i}, i \Delta t \mid z_{i-1},(i-1) \Delta t\right) \approx \delta\left(z_{i}-\bar{z}\right)$ inside the integrals (9), we would neglect the role of the volatility and consider only a drift growth of the asset prices. In order to take care of the volatility effects, a possible solution is to estimate the integral of interest, i.e.

$$
E\left[\mathcal{O}_{i} \mid S_{i-1}\right]=\int_{-\infty}^{+\infty} d z p\left(z \mid z_{i-1}\right) \mathcal{O}_{i}\left(e^{z}\right)
$$

by inserting in eq. (26) the analytical expression for the $p\left(z \mid z_{i-1}\right)$ transition probability

$$
\begin{aligned}
p\left(z \mid z_{i-1}\right)= & \frac{1}{\sqrt{2 \pi \Delta t \sigma^{2}}} \exp \left\{-\frac{\left(z-z_{i-1}-A \Delta t\right)^{2}}{2 \sigma^{2} \Delta t}\right\}= \\
& \frac{1}{\sqrt{2 \pi \Delta t \sigma^{2}}} \exp \left\{-\frac{(z-\bar{z})^{2}}{2 \sigma^{2} \Delta t}\right\},
\end{aligned}
$$

together with a Taylor expansion of the kernel function $\mathcal{O}_{i}\left(e^{z}\right) \doteq f(z)$ around the expected value $\bar{z}$. Hence, up to the second order in $z-\bar{z}$, the kernel function becomes

$$
f(z)=f(\bar{z})+(z-\bar{z}) f^{\prime}(\bar{z})+\frac{1}{2} f^{\prime \prime}(\bar{z})(z-\bar{z})^{2}+O\left((z-\bar{z})^{3}\right),
$$

which, together with eq. (5), yields

$$
E\left[\mathcal{O}_{i} \mid S_{i-1}\right]=f(\bar{z})+\frac{\sigma^{2}}{2} f^{\prime \prime}(\bar{z}),+\ldots
$$

since the first derivative does not give contribution to eq. (26), being the integral of an odd function over the whole $z$ range. The second derivative can be numerically estimated as

$$
f^{\prime \prime}(\bar{z})=\frac{1}{\delta_{\sigma}^{2}}\left[f\left(\bar{z}+\delta_{\sigma}\right)-2 f(\bar{z})+f\left(\bar{z}-\delta_{\sigma}\right)\right],
$$

with $\delta_{\sigma}=O(\sigma \sqrt{\Delta t})$, as dictated by the dynamics of the stochastic process. It is worth noticing that each expectation value $E\left[\mathcal{O}_{i} \mid S_{i-1}\right]$ can be now computed once $f(\bar{z})=\mathcal{O}_{i}\left(e^{z_{i-1}+A \Delta t}\right)$ and $f\left(\bar{z} \pm \delta_{\sigma}\right)=\mathcal{O}_{i}\left(e^{z_{i-1}+A \Delta t \pm \delta_{\sigma}}\right)$ are known. Consequently, if we employ the deterministic grid illustrated in Section 3.2, it 
Table 3

Price of an American put option with $t=0$ year, $T=0.5$ year, $r=0.1, \sigma=0.4$, $X=10$, as a function different stock prices $S_{0}$. The path integral results are obtained with $\delta_{\sigma}=2 \sigma \sqrt{\Delta t}, 300$ time slices and $p=3$.

\begin{tabular}{ccccc}
\hline \hline$S_{0}$ & finite difference & binomial tree & GFDNM & path integral \\
\hline \hline 6.0 & 4.00 & 4.00 & 4.00 & 4.00 \\
8.0 & 2.095 & 2.096 & 2.093 & 2.095 \\
10.0 & 0.921 & 0.920 & 0.922 & 0.922 \\
12.0 & 0.362 & 0.365 & 0.364 & 0.362 \\
14.0 & 0.132 & 0.133 & 0.133 & 0.132 \\
\hline \hline
\end{tabular}

is enough to put $p=3$ to obtain reliable results, provided $\Delta t$ is taken sufficiently small. Actually, the results obtained with this simple semi-analytical procedure are shown in Tab. 3, using $\delta_{\sigma}=2 \sigma \sqrt{\Delta t}$ for numerical differentiation and 300 time slices. For such path integral evaluation of an American option the CPU time is negligible. The results are in nice agreement with those of other numerical procedures and in perfect agreement, as we explicitly checked for different model parameters, with those quoted in Tab. 2 as obtained with the path integral algorithm discussed in Section 3.2.

\section{Concluding remarks and outlook}

In this paper we have shown that the path integral approach to stochastic processes can be successfully applied to the problem of option pricing in financial analysis. In particular, an efficient implementation of the path integral method has been presented, in order to obtain fast and accurate predictions for a large class of financial derivatives, including those with path-dependent and early exercise features. The key points of the algorithm are a careful evaluation of the transition probability associated to the stochastic model for the time evolution of the asset prices and a suitable choice of the integration points needed to evaluate the quantities of financial interest. Furthermore, a simple and very fast procedure to value American options has been derived, by exploiting the possibility of continuous exercise up to the expiration date.

The results of the path integral algorithm have been carefully compared with those available in the literature for European and American options in the BSM model and found to be in good agreement with the standard numerical procedures used in finance. The computational time of the algorithm developed is, to the best of our knowledge, competitive with the most efficient strategies used in finance. The method is general and it can be quite easily extended to cope with other financial derivatives (with path-dependent features) 
and other models beyond the dynamics of geometric Brownian motion.

The natural developments of the path integral algorithm here presented concern the application of the method to value other kinds of quantities of financial interest, for which the analytical solution is not available or not accessible, and the extension of the method of option pricing to more realistic model of the financial dynamics, such as models with stochastic volatility $[3,12,18]$ or beyond the BSM Gaussian limit [19-24], in order to search for a better agreement with the real prices as observed in the real market. A further interesting perspective would be using the path integral algorithm as a benchmark to train neural networks.

Such developments are by now under consideration.

\section{Acknowledgments}

We acknowledge collaboration of F. Piccinini at the early stage of this work. We wish to thank M. Cersich, E. Melchioni and A. Pallavicini of FMR Consulting for useful discussions. We are also grateful to FMR Consulting for having provided the software for the analytical predictions of the price and Greeks of European options.

\section{References}

[1] F. Black and M. Scholes, Journal of Political Economics 72 (1973) 637.

[2] R. Merton, Bell J. Econom. Managem. Sci. 4 (1973) 141.

[3] J.C. Hull, Options, Futures, and Other Derivatives, Fourth Edition, Prentice Hall, New Jersey, 2000.

[4] W. Paul and J. Baschnagel, Stochastic Processes: from Physics to Finance, Springer-Verlag, Berlin Heidelberg, 1999.

[5] P. Wilmott, J. Dewynne and S. Howinson, Option Pricing: Mathematical Models and Computation, Oxford Financial Press, Oxford, 1993.

[6] M. Potters, J.-P. Bouchaud and D. Sestovic, Physica A 289 (2001) 517.

[7] N. Wiener, Proc. Nat. Acad. Sci. (USA) 7 (1952) 253; Proc. Nat. Acad. Sci. (USA) 7 (1952) 294.

[8] M. Kac, Bull. Amer. Math. Soc. 72 (1966) 52.

[9] R. P. Feynman, Rev. Mod. Phys. 20 (1948) 367;

R. P. Feynman and A. R. Hibbs, Quantum Mechanics and Path Integral, McGraw-Hill, New York, 1965. 
[10] E. Bennati, M. Rosa-Clot and S. Taddei, A Path Integral Approach to Derivative Security Pricing: I. Formalism and Analytical Results, Int. Journ. Theor. Appl. Finance 2 (1999) 381, cond-mat/9901277.

[11] M. Rosa-Clot and S.Taddei, A Path Integral Approach to Derivative Security Pricing: II. Numerical Methods, cond-mat/9901279.

[12] B.E. Baaquie, J. Phys. I France 7 (1997) 1733.

[13] L. Ingber, High-Resolution Path-Integral Development of Financial Options, physics/0001048.

[14] A. Matacz, Path Dependent Option Pricing: the Path Integral Partial Averaging Method, cond-mat/0005319.

[15] L.S. Schulman, Techniques and Applications of Path Integration, John Wiley \& Sons, New York, 1981.

[16] W.H. Press, B.P. Flannery, S.A. Teukolsky and W.T. Vetterling, Numerical Recipes - The Art of Scientific Computing, Cambridge University Press, New York, 1989.

[17] NAG Fortran library, The Numerical Algorithms Group Ltd, Oxford.

[18] J.C. Hull and A. White, Journal of Finance 42 (1987) 281;

J. Masoliver and J. Perello, A Correlated Stochastic Volatility Model Measuring Leverage and Other Stylized Facts, cond-mat/0111334.

[19] R.N. Mantegna and H.E. Stanley, An Introduction to Econophysics: Correlations and Complexity in Finance, Cambridge University Press, Cambridge, 2000.

[20] J.P. Bouchaud and M. Potters, Theory of Financial Risk: from Statistical Physics to Risk Management, Cambridge University Press, Cambridge, 2000.

[21] B.B. Mandelbrot, Fractals and Scaling in Finance, Springer-Verlag, Berlin Heidelberg, 1997.

[22] R.N. Mantegna and H.E. Stanley, Phys. Rev. Lett. 73 (1994) 2946;

R.N. Mantegna and H.E. Stanley, Nature 383 (1996) 587;

R.N. Mantegna, Z. Palágyi and H.E. Stanley, Physica A 274 (1999) 216.

[23] A. Matacz, Financial Modeling and Option Theory with the Truncated Levy Process, cond-mat/9710197.

[24] Frederick Michael and M.D. Johnson, Financial Market Dynamics, cond-mat/0108017. 